\title{
Introducción de ganado europeo en la Puna jujeña y su rol en las economías tradicionales. Zooarqueología del sitio histórico Antigal Laguna
}

\section{Julia Merler Carbajo*}

Fecha de defensa: 22 de diciembre de 2020

Director: Dr. Hugo D. Yacobaccio

Jurados: Dres. Matilde Lanza y Gabriel López

La problemática abordada en esta tesis corresponde a cómo se dio el proceso de incorporación de fauna europea doméstica en las economías locales de la Puna jujeña durante los momentos posteriores a la conquista. De acuerdo con lo planteado por las fuentes históricas, los animales domésticos introducidos por los conquistadores se dispersaron rápidamente por los territorios americanos (Crosby, 1986). En el caso del Noroeste Argentino (NOA), estas especies se habrían incorporado de manera relativamente fácil a la cotidianeidad de las poblaciones nativas que vivían en la región y habrían cumplido un rol clave dentro del sistema económico y social impuesto por los españoles, el cual se estructuraba sobre tres pilares: minería, ganadería y comercio (Gil Montero, 2009; Palomeque, 2000; Sanhueza, 1992, entre otros).

En la región de la Puna, la preexistencia de una extensa red de rutas de intercambio mediante caravanas de llamas y el hecho de que el pastoreo se estructurara como actividad económica principal durante momentos prehispánicos y, por ende, que existiera una base de experiencia en el manejo de animales de rebaño, determinó que la incorporación de la fauna doméstica europea se diera con mayor facilidad que en otras regiones y favoreció la participación indígena en el nuevo sistema económico (Gil Montero, 2009).

Sin embargo, la disponibilidad de fuentes y documentos históricos que hagan referencia al proceso de incorporación de estas especies en la Puna es escasa. Esto se debe principalmente a la condición de aislamiento de la región de los centros de poder (Vaquer, 2016), su poca accesibilidad y la existencia de un clima árido y poco apto para el desarrollo agrícola. Esto determinó que la ocupación de la región se diera de manera más tardía y que el control colonial fuera menor que en otras áreas del NOA (Aramendi, 2016; Gil Montero, 2009).

Respecto a las investigaciones arqueológicas, los sitios que cuentan con fauna europea dan cuenta de su presencia en una gran diversidad de contextos, ya sea domésticos (Nielsen, Angiorama, Maryñanski, Ávila y Lopez, 2015; Quiroga, 2015), vinculados con la minería (Angiorama, Becerra y Pérez Pieroni,

* Instituto de Arqueología, Facultad de Filosofía y Letras, Universidad de Buenos Aires (UBA). 25 de mayo 217, $3^{\circ}$ piso (CP C1002ABE) Ciudad Autónoma de Buenos Aires, Argentina. E-mail: julimerler@yahoo.com.ar
2015; Vaquer, 2016), rituales (Capparelli, Lema, Giovannetti y Raffino, 2005), en entierros (Otero y Rivolta, 2015) o como materia prima (Von Rosen, 1957). Sin embargo, los sitios que presentan esta fauna en el registro son pocos, a lo que se suma el hecho de que no hay muchos trabajos arqueológicos que aborden el proceso de introducción de estas especies en las economías de pequeña escala de la Puna.

Es así que el objetivo principal de este trabajo fue el de indagar en el impacto producido por las especies domésticas europeas al ser incorporadas a los rebaños de las poblaciones que habitaron la Puna jujeña, más específicamente el valle del río Barrancas, entre los siglos XVII a XVIII, a partir del estudio de los restos faunísticos provenientes del sitio histórico Antigal Laguna. Se buscó conocer cuáles fueron las estrategias de manejo para las distintas especies y cómo éstas se diferencian de las utilizadas para los rebaños de camélidos, cuáles fueron los usos y para qué fueron destinadas, con el objetivo de evaluar cómo estos animales se incorporaron a la vida cotidiana de los pastores y de qué manera afectaron su participación en el contexto político y económico instaurado durante la conquista europea. Para abordar estos interrogantes se plantearon los siguientes objetivos específicos:

a. Determinar la composición taxonómica y anatómica de los conjuntos faunísticos.

b. Evaluar la integridad de los conjuntos de manera de poder conocer el grado de alteración producida por agentes tafonómicos, tanto bióticos como abióticos.

c. Determinar la asociación de los conjuntos con actividades humanas y evaluar los patrones de obtención, procesamiento, consumo y descarte implementados para cada especie.

d. Reconocer los rangos etarios presentes para cada especie, de manera de poder identificar los patrones de matanza a los que estuvieron sujetas.

El sitio arqueológico Antigal Laguna se encuentra en la Localidad Arqueológica de Barrancas, ubicada en el valle del río homónimo, en el departamento de Cochinoca, provincia de Jujuy. La localidad presenta cuatro sitios que dan cuenta de la presencia de ocupaciones durante momentos post hispánicos. En estos sitios se hallaron restos de fauna europea, cuentas de vidrio, fragmentos de vidrio y mayólica, así como paneles con motivos rupestres de jinetes, caballos y cruces cristianas. La 
mayoría cuentan, además, con fechados entre los siglos XVI y XVIII (Yacobaccio, 2014).

La muestra proviene de las prospecciones subsuperficiales realizadas en el sector norte del sitio, el cual está compuesto por tres estructuras, dos corrales y cuatro trojas o depósitos distribuidos a lo largo de 150 metros lineales sobre una terraza estructural. Se realizaron dos sondeos de $1 \mathrm{~m}^{2}$ dentro y fuera de la estructura 3 , adyacentes a la pared sudoeste de la misma, de los cuales se obtuvo un total de 1.714 especímenes óseos.

Los pasos metodológicos que involucró el análisis del material incluyeron, en primer lugar, la identificación taxonómica y anatómica de los especímenes, a partir de la utilización de manuales osteológicos (Adams y Crabtree, 2012; Hopkins, Hamm y Leppart, 1970; Pacheco Torres, Altamiro y Guerras Porras, 1986). Sobre esta base se obtuvieron distintas medidas de cuantificación tales como el Número de Especímenes Identificados por Taxón (NISP), Mínimo Número de Elementos (MNE), Mínimo Número de Unidades Anatómicas (MAU y MAU calculado en porcentaje) y el Mínimo Número de Individuos (MNI) (Lyman, 1994; Mengoni Goñalons, 1999). A su vez se calcularon los grupos etarios representados a partir de la erupción y desgaste dentario (Wheeler, 1982) y de la fusión de las epífisis (Mengoni Goñalons, 1999) para los camélidos y para el género Bos (Hongo, 1998).

En segundo lugar, se abordaron variables que permitieran evaluar la incidencia de agentes tafonómicos sobre los especímenes. Se midió la meteorización (Behrensmeyer, 1978) y se consideró la presencia de marcas de carnívoro, roedores y raíces sobre la superficie ósea (Binford, 1981; Lyman, 1994; Mengoni Goñalons, 1999), así como la depositación de manganeso (Barrientos et al., 2007) y carbonato de calcio (Cornaglia Fernandez, 2012).

En tercer lugar, para evaluar la asociación de los conjuntos con actividades antrópicas, se consideró la presencia, distribución y orientación de marcas antrópicas (Binford, 1981), la presencia de fractura transversal, espiral y longitudinal (Mengoni Goñalons, 1980) y la presencia de termoalteración (Mengoni Goñalons, 1999).

Por último, se apeló al uso de modelos etnoarqueológicos de pastoreo andino como marcos de referencia que permitieran derivar hipótesis sobre el tipo de manejo utilizado tanto para los camélidos como para las especies europeas, dado que, actualmente, éstas se encuentran completamente incorporadas a los rebaños de la Puna (Madero, 2000; Yacobaccio, 2007, entre otros).

Los resultados obtenidos muestran un amplio predominio del taxón Camelidae (53,5\%). Las marcas antrópicas identificadas en los especímenes estarían indicando actividades de cuereo y de carneo realizadas en el sitio. Esto se sustenta por la presencia de los esqueletos completos y una gran cantidad de individuos (MNI de 18). La existencia de fracturas podría vincularse a actividades de origen humano, aunque no se descarta su relación con la acción de carnívoros. La presencia de restos termoalterados permite pensar, además, que se trata de restos descartados dentro de un fogón dentro de la estructura. Por último, el predominio de individuos jóvenes y neonatos por sobre los adultos y viejos podría dar cuenta de estrategias de matanza destinadas a la producción de carne y, en menor medida, de transporte y lana (Madero, 2000; Yacobaccio, 2007).

Las especies europeas se encuentranen porcentajes muy bajos. Se identificaron cuatro especímenes de ovicápridos y 32 de Bos (de los cuales 29 corresponden a fragmentos de diente). Ambos taxones presentan evidencias de acción antrópica. En el caso de los ovicápridos, no se observaron fracturas, pero sí marcas de corte y termoalteración. Dada la intensidad de marcas de carnívoro identificada en dos especímenes, no se puede descartar que la presencia de estos animales en el registro se encuentre sesgada por la acción de estos animales. En el caso de Bos, se identificó únicamente fractura y la existencia de un fragmento de metapodio perteneciente a un individuo joven o novillo, lo que indicaría un posible consumo de carne.

Resaltan en la muestra tres especímenes de Lagidium viscacia con marcas antrópicas, fractura y termoalteración. Dado que el consumo de estos animales en la Puna se encuentra documentado desde el Holoceno temprano y continuó luego de la domesticación de camélidos, es posible su presencia en Antigal Laguna esté vinculada a un encuentro fortuito de caza y consumo.

A grandes rasgos, los patrones observados en el registro faunístico de Antigal Laguna se condicen con el panorama planteado por las fuentes históricas y con lo observado en otros sitios arqueológicos a nivel regional. Las proporciones en las que se encuentran representados los camélidos en Antigal Laguna reflejan los patrones esperables en sitios arqueológicos pertenecientes a grupos de pastores en esta región, en los cuales suele predominar el taxón Camelidae, en detrimento de los ovicápridos. En el caso de estos últimos, es posible que su presencia esté vinculada a un consumo ocasional, dada su poca representación.

En el caso de las vacas, las fuentes las vinculan mayoritariamente al comercio y la arriería mediante la cual eran trasladadas para abastecer los centros mineros. EI registro arqueológico regional refleja en parte esta situación en sitios vinculados a la actividad minera y con presencia de restos óseos de Bos. Sin embargo, hay algunos sitios en los que estos animales aparecen en contextos domésticos y de consumo familiar, más que relacionados con actividades comerciales o productivas. Este parecería ser el caso de Antigal Laguna, donde las vacas habrían sido producto de un consumo doméstico y ocasional. 
Como fue mencionado anteriormente, la información disponible acerca del ingreso de las especies europeas domésticas en las economías pastoriles de la Puna, el manejo que se le dio a cada una y los roles que cumplieron, es muy escasa. Sin embargo, los datos disponibles hasta ahora constituyen un punto de partida sumamente importante para responder a estas preguntas y permiten plantear un panorama preliminar sobre el proceso de incorporación de estas especies en el pastoreo andino y cómo éste se desarrolló durante momentos posteriores a la conquista europea.

\section{Referencias citadas}

" Adams, B. y Crabtree, P. (2012). Comparative Osteology. A Laboratory and Field Guide of Common North American Animals. Nueva York: Academic Press.

» Angiorama, C., Becerra, M. F. y Pérez Pieroni, M. J. (2015). El mineral de Pan de Azúcar. Arqueología histórica de un centro minero colonial en la Puna de Jujuy (Argentina). Chungara, Revista de Antropología Chilena, 47(4), 603-619. http://dx.doi.org/10.4067/S0717-73562015005000045

" Aramendi, B. (2016). El distrito de la Puna y su primer subdelegado. Intendencia de Salta del Tucumán, 17841795. Fronteras de La Historia, 22(1), 12-37.

» Barrientos, G., Goñi, R., Zangrando, A., Del Papa, M., García Guraieb, S., Arregui, M. J. y Negro, C. (2007). Human taphonomy in Southern Patagonia: a view from the Salitroso Lake Basin (Santa Cruz, Argentina). Taphonomy and Zooarchaeology in Argentina, 1601(1426), 187-201.

"Behrensmeyer, A. K. (1978). Taphonomic and ecologic information from bone weathering. Paleobiology, 4(2), 150-162.

" Binford, L. R. (1981). Bones. Ancient Men and Modern Myths. Orlando: Academic Press.

》 Capparelli, A., Lema, V., Giovannetti, M. y Raffino, R. (2005). The introduction of Old World crops (wheat, barley and peach) in Andean Argentina during the 16th century AD: archaeobotanical and ethnohistorical evidence. Vegetation History and Archaeobotany, 14(4), 472-484. https://doi. org/10.1007/s00334-005-0093-8.

" Cornaglia Fernandez, J. (2012). El carbonato de calcio y sus implicancias en el análisis de los conjuntos arquefaunísticos. El caso de Laguna El Doce (Departamento General Lopez, Provincia de Santa Fe). Revista del Museo de Antropologia, 5(1), 185-194. https:// doi.org/10.31048/1852.4826.v5.n1.9138.

"Crosby, A. W. (1986). Ecological Imperialism. The Biological Expansion of Europe, 900 - 1900. Nueva York: Cambridge University Press.

》 Gil Montero, R. (2009). Mountain pastoralism in the Andes during colonial times. Nomadic Peoples, 13(2), 36-50. https://dx.doi.org/10.3167/np.2009.130203.

» Hongo, H. (1998). Patterns of animal husbandry in central Anatolia in the second and first millenia BC: Faunal remains from Kaman-kalehöyük, Turkey. En H. Buitenhuis, L. Bartosiewicz y A. M. Choyke (Eds.), Achaeozoology of the Near East III. Procedings of the Third International
Symposium on the Archaeological of Southwestern Asia and Adjacent Areas (pp. 255-275). Groningen: ARC (Publicatie 18).

» Hopkins, C. E., Hamm, T. E. y Leppart, G. L. (1970). Atlas of Goat anatomy. Part l: Osteology. Maryland: Edgewood Arsenal Department of the Army.

» Lyman, R. L. (1994). Vertebrate Taphonomy. Cambridge: Cambridge University Press.

» Madero, C. M. (2000). Modelo etnoarqueológico de estructura etaria en contextos pastoriles actuales de la Puna de Jujuy. Cuadernos del Instituto Nacional de Antropología y Pensamiento Latinoamericano, 19, 285-399.

» Mengoni Goñalons, G. L. (1980). Notas zooarqueológicas I: Fractura en huesos. En Actas del VII Congreso Nacional de Arqueología (pp. 87-91). Montevideo: Centro de Estudios de Arqueología.

» Mengoni Goñalons, G. L. (1999). Cazadores de Guanacos de la Estepa Patagónica. Buenos Aires: Sociedad Argentina de Antropología.

» Nielsen, A. E., Angiorama, C., Maryñanski, J., Avila, F. y López, M. L. (2015). Paisajes prehispánicos tardíos en San Juan Mayo (Frontera Argentina-Bolivia). Arqueología, 21, 33-65.

» Otero, C. y Rivolta, M. C. (2015). Nuevas interpretaciones para la secuencia de ocupación de Tilcara (Quebrada de Humahuaca, Jujuy). Intersecciones en Antropologia, 16(1), 145-159.

» Pacheco Torres, V. R., Altamiro, A. J. y Guerra Porras, E. S. (1986). The Osteology of South American Camelids. Los Angeles: Institute of Archaeology, University of California.

» Palomeque, S. (2000). El mundo indígena (siglos XVI-XVII). Historia Colonial, 2, 87-145.

» Quiroga, L. (2015). Del páramo a la Puna. Textos y contextos arqueológicos para una descripción del paisaje altoandino en la gobernación del Tucumán. Corpus. Archivos Virtuales de la Alteridad Americana, 5(2). https://doi.org/10.4000/ corpusarchivos.1516.

»Sanhueza, M. C. (1992). Tráfico caravanero y arriería colonial en el siglo XVI. Estudios Atacameños, 10, 169-182.

»Vaquer, J. M. (2016). La ocupación colonial temprana (S . XVI y XVII) en Casas Quemadas (Cusi Cusi, Rinconada, Jujuy): primeras aproximaciones a las relaciones entre lo global y lo local. Revista de Arqueología Histórica Argentina y Latinoamericana, 10(2), 1-26.

»Von Rosen, E. (1957). Restos de una cultura indígena. En Un Mundo que se Va (pp. 87-125). San Miguel de Tucumán: Instituto Miguel Lillo, Universidad Nacional de Tucumán.

»Wheeler, J. C. (1982). Aging llamas and alpacas by their teeth. Llama World, 1(2), 12-17.

» Yacobaccio, H. D. (2007). Andean camelid herding in the South Andes: Ethnoarchaeological models for archaeozoological research. Anthropozoologica, 42(2), 143-154.

» Yacobaccio, H. D. (2014). Arqueología ambiental, arte rupestre y gestión del patrimonio cultural en Barrancas (Abdón Castro Tolay, Puna de Jujuy). Informe a la Comisión Municipal de Barrancas. Manuscrito inédito. 